\title{
ASYMPTOTICALLY NORMAL ESTIMATION OF A PARAMETER IN A LINEAR-FRACTIONAL REGRESSION PROBLEM
}

\author{
YU. YU. LINKE AND A. I. SAKHANENKO
}

\section{INTRODUCTION}

Suppose that in some experiment we observe a sequence of independent random variables $X_{1}, X_{2}, \ldots, X_{N}$ such that the following representation is valid for every $i$ :

$$
X_{i}=\frac{a_{i}}{1+b_{i} \theta}+\sigma_{i} \xi_{i},
$$

with $\xi_{1}, \ldots, \xi_{N}$ a sequence of independent identically distributed random variables satisfying the conditions

$$
\mathbf{E} \xi_{i}=0, \quad \mathbf{D} \xi_{i}=1
$$

Moreover, the values $a_{i}>0$ and $b_{i}>0$ are assumed known, while the values of the parameter $\theta$ and the variances $\mathbf{D} X_{i} \equiv \sigma_{i}^{2}$ are unknown. The values of the random variables $\xi_{1}, \ldots, \xi_{N}$ are assumed unknown either.

In this article, we study the problem of estimating the unknown parameter $\theta>0$ from the observations $X_{1}, \ldots, X_{N}$. This problem is a particular instance of the nonlinear regression problem which is usually solved by the method of least squares or its modifications. Searching an estimator approximately, we often use linearization methods, the steepest descent method, etc. (see, for instance, [1]) whose implementation requires application of computers in view of a huge number of iterations.

However, it turns out that for a linear-fractional regression problem of the form (1.1) the simple estimator

$$
\theta^{*}=\frac{\sum c_{i}\left(a_{i}-X_{i}\right)}{\sum c_{i} b_{i} X_{i}}
$$

is asymptotically normal under rather general assumptions on the constants $\left\{c_{i}\right\}$. Moreover, in the case when some information on the behavior of the variances $\left\{\sigma_{i}\right\}$ is available we can choose functions $\left\{\gamma_{i}(\theta)\right\}$ so that the "improved" estimator

$$
\theta^{* *}=\frac{\sum \gamma_{i}\left(\theta^{*}\right)\left(a_{i}-X_{i}\right)}{\sum \gamma_{i}\left(\theta^{*}\right) b_{i} X_{i}}
$$

becomes asymptotically efficient in some sense.

The present article is devoted to constructing a class of these two-step estimators and studying their properties. The main results reside in $\S 2$ in which, for clarity of exposition, we do not pursue the goal of giving most general statements. In

Date: submitted June 27, 1997. 
a more abstract situation, the corresponding assertions are given in $\S 3$ and proven in $\S 4-\S 6$.

We note that the methods of the article extend to the case of a multidimensional parameter when the following representation is valid:

$$
X_{i}=\frac{a_{i}^{0}+\sum_{j=1}^{k} a_{i}^{j} \theta_{j}}{1+\sum_{j=1}^{k} b_{i}^{j} \theta_{j}}+\sigma_{i} \xi_{i} .
$$

In particular, we can construct estimators for unknown parameters in the MichaelisMenten equation which is widely used in biochemistry (see, for instance, [2]). These results will be given in the forthcoming articles, wherein (1.1) will appear as a particular case of (1.5) enabling us to illustrate all ideas of our method without obscuring them by bulky matrix notations.

We use the symbol $\sum$ without indices only when summation is carried out over $i$ from 1 to $N$. Below, unless the contrary is specified, we consider all limits as $N \rightarrow \infty$ and use the notation $\Phi(x)=(2 \pi)^{-1 / 2} \int_{-\infty}^{x} e^{-y^{2} / 2} d y$ for the distribution function of the standard normal law.

\section{The Main Results}

In this section, we study the properties of the estimators (1.3) and (1.4) in the case when the following easy conditions are met:

$$
\inf _{i} \min \left\{a_{i}, b_{i}, c_{i}, \sigma_{i}\right\}>0, \quad \sup _{i} \max \left\{a_{i}, b_{i}, c_{i}, \sigma_{i}\right\}<\infty
$$

Put

$$
d^{2}\left(\left\{c_{i}\right\}\right)=d_{N, \theta}^{2}\left(\left\{c_{i}\right\},\left\{\sigma_{i}\right\}\right)=\frac{\sum c_{i}^{2}\left(1+b_{i} \theta\right)^{2} \sigma_{i}^{2}}{\left(\sum c_{i} a_{i} b_{i}\left(1+b_{i} \theta\right)^{-1}\right)^{2}} .
$$

Theorem 1. If (2.1) is satisfied then

$$
\sup _{x}\left|\mathbf{P}\left(\frac{\theta^{*}-\theta}{d\left(\left\{c_{i}\right\}\right)}<x\right)-\Phi(x)\right| \rightarrow 0 .
$$

Now, consider the behavior of a more complicated estimator $\theta^{* *}$ given by (1.4). Throughout the article, we suppose that all functions $\left\{\gamma_{i}(\theta)\right\}$ are differentiable with respect to $\theta$ and the derivatives $\gamma_{i}^{\prime}(\theta)$ satisfy the condition

$$
\sup _{\theta / 2 \leq t \leq 2 \theta}\left|\gamma_{i}^{\prime}(t)\right| \leq K_{i}(\theta)<\infty .
$$

Theorem 2. Suppose that (2.1) is satisfied and the functions $\left\{\gamma_{i}(\theta)\right\}$ are such that

Then

$$
\inf _{i} \gamma_{i}(\theta)>0, \quad \sup _{i}\left(\gamma_{i}(\theta)+K_{i}(\theta)\right)<\infty
$$

$$
\sup _{x}\left|\mathbf{P}\left(\frac{\theta^{* *}-\theta}{d\left(\left\{\gamma_{i}(\theta)\right\}\right)}<x\right)-\Phi(x)\right| \rightarrow 0,
$$

where $d\left(\left\{\gamma_{i}(\theta)\right\}\right)$ is determined by (2.2) with $c_{i}=\gamma_{i}(\theta)$.

Remark 1. It is clear that the accuracy of the estimators $\theta^{*}$ and $\theta^{* *}$ is determined by the coefficients $d^{2}\left(\left\{c_{i}\right\}\right)$ and $d^{2}\left(\left\{\gamma_{i}(\theta)\right\}\right)$. Therefore, the problem appears 
naturally of minimizing these coefficients. The following equalities are easy to verify for every $C>0$ :

$$
d_{\mathrm{opt}}^{2} \equiv \inf _{\left\{c_{i}\right\}} d^{2}\left(\left\{c_{i}\right\}\right)=\inf _{\left\{\gamma_{i}(\theta)\right\}} d^{2}\left(\left\{\gamma_{i}(\theta)\right\}\right)=d^{2}\left(\left\{\gamma_{\mathrm{opt}, i}\left(\theta, \sigma_{i}\right)\right\}\right),
$$

with

$$
\gamma_{\mathrm{opt}, i}\left(\theta, \sigma_{i}\right)=C \frac{a_{i} b_{i}}{\left(1+b_{i} \theta\right)^{3} \sigma_{i}^{2}} .
$$

We emphasize that $C$ in (2.7) may be an arbitrary positive parameter independent of $i$.

Remark 2. Suppose that the independent random variables $\xi_{i}$ have the standard normal distribution. Then the variables $X_{i}$ are normally distributed with mean $U_{i}(\theta)=a_{i} /\left(1+b_{i} \theta\right)$ and variance $\sigma_{i}^{2}$. Suppose that the variances $\sigma_{i}^{2}$ are independent of $\theta$. In this case

$$
I_{N}(\theta)=\sum \frac{\left(U_{i}^{\prime}(\theta)\right)^{2}}{\sigma_{i}^{2}}=\sum \frac{a_{i}^{2} b_{i}^{2}}{\sigma_{i}^{2}\left(1+b_{i} \theta\right)^{4}},
$$

where $I_{N}(\theta)$ is the Fisher information for the sample $X_{1}, \ldots, X_{N}$. Inserting the optimal value $\gamma_{i}(\theta)=\gamma_{\mathrm{opt}, i}\left(\theta, \sigma_{i}\right)$ of Remark 1 in the coefficient of the asymptotic variance and using (2.5), we obtain

$$
d_{\mathrm{opt}}^{2}=1 / I_{N}(\theta) .
$$

Relation (2.6) shows that, by analogy with the Cramér-Ráo inequality, we should expect the estimator $\theta^{* *}$ to be in a sense unimprovable when $\gamma_{i}(\theta)$ are chosen optimally.

Example 1. Suppose that

$$
\sigma_{i}^{2}=w_{o i}\left(1+b_{i} \theta\right)^{-3} \sigma^{2},
$$

where the coefficient $w_{o i}>0$ is assumed to be known, while the parameter $\sigma>0$ may be unknown. Then, by Remark 1 on Theorem 1, we can choose optimal constants $c_{i}$ by putting $c_{i}=a_{i} b_{i} / w_{o i}$.

Remark 3. Using Remark 1, we can easily verify that Example 1 gives the only case in which the optimal values $c_{i}$ are constants rather than functions of $\theta$ and $\sigma_{i}$.

Example 2. Suppose that

$$
\sigma_{i}^{2}=\sigma^{2} w_{i}(\theta),
$$

where $w_{i}(\theta)$ are known functions and the parameter $\sigma>0$ may be unknown. It is easy to see that in this case we can put

$$
\gamma_{\mathrm{opt}, i}\left(\theta, \sigma_{i}\right)=\gamma_{\mathrm{opt}, i}(\theta) \equiv \frac{a_{i} b_{i}}{\left(1+b_{i} \theta\right)^{3} w_{i}(\theta)} .
$$

Now, suppose that all conditions of Theorem 2 are satisfied for

$$
\gamma_{i}(\theta)=\gamma_{\mathrm{opt}, i}(\theta), \quad c_{i}=\gamma_{\mathrm{opt}, i}\left(\theta_{0}\right),
$$

where $\theta_{0}$ is some fixed value of $\theta$. Then the conclusion of Theorem 2 holds; moreover, $d^{2}\left(\left\{\gamma_{i}(\theta)\right\}\right)=d_{\mathrm{opt}}^{2}$.

Thus, in the case of Example 2 we can recommend using the estimators $\theta^{*}$ and $\theta^{* *}$ for $c_{i}$ and $\gamma_{i}(\theta)$ in (2.7). Moreover, the estimator $\theta^{* *}$ of the second step is asymptotically normal with the asymptotic variance $d_{\mathrm{opt}}^{2}$. 
Example 3 Suppose that $\sigma_{i}^{2}=\sigma^{2}$, where $\sigma>0$ is an unknown parameter. By analogy with Example 2, in this case we can recommend using the estimators $\theta^{*}$ and $\theta^{* *}$ for $c_{i}=a_{i} b_{i}$ and $\gamma_{i}(\theta)=\gamma_{\text {opt }, i}(\theta) \equiv a_{i} b_{i} /\left(1+b_{i} \theta\right)^{3}$.

Remark 4. If the exact form of the variance $\sigma_{i}$ is unknown then we cannot find $\gamma_{\mathrm{opt}, i}\left(\theta, \sigma_{i}\right)$ and construct the estimator $\theta^{* *}$ for $\gamma_{i}(\theta)=\gamma_{\mathrm{opt}, i}\left(\theta, \sigma_{i}\right)$. In this case we can recommend taking $\gamma_{i}(\theta)$ to be functions that may be assumed to "differ slightly" from the unknown functions $\gamma_{\mathrm{opt}, i}\left(\theta, \sigma_{i}\right)$.

Remark 5. It is easy to verify that

$$
1 \leq \frac{d^{2}\left(\left\{\gamma_{i}(\theta)\right\}\right)}{d_{\mathrm{opt}}^{2}} \leq \frac{\sup _{i \leq N}\left(\gamma_{i}(\theta) / \gamma_{\mathrm{opt}, i}\left(\theta, \sigma_{i}\right)\right)}{\inf _{i \leq N}\left(\gamma_{i}(\theta) / \gamma_{\mathrm{opt}, i}\left(\theta, \sigma_{i}\right)\right)} ;
$$

i.e., the "better" the chosen functions $\gamma_{i}(\theta)$ approximate the functions $\gamma_{\mathrm{opt}, i}\left(\theta, \sigma_{i}\right)$, the less the asymptotic variance of the corresponding estimator differs from $d_{\mathrm{opt}}^{2}$. We can treat (2.8) as some stability property for the estimators $\theta^{* *}$ as functionals depending on the functions $\gamma_{i}(\theta)$.

In constructing the confidence intervals and test of hypotheses, it would be more convenient to have analogs of Theorems 1 and 2 in which the parameters $d\left(\left\{c_{i}\right\}\right)$ and $d\left(\left\{\gamma_{i}(\theta)\right\}\right)$ are replaced with some their estimators. We give assertions which possess these properties. Put

$$
\begin{gathered}
\beta_{i}^{*}=\left(1+b_{i} \theta^{*}\right) X_{i}-a_{i}, d^{*}=\left(\sum c_{i}^{2} \beta_{i}^{* 2}\right)^{1 / 2} / \sum c_{i} b_{i} X_{i}, \\
d^{* *}=\left(\sum \gamma_{i}^{2}\left(\theta^{*}\right) \beta_{i}^{* 2}\right)^{1 / 2} / \sum \gamma_{i}\left(\theta^{*}\right) b_{i} X_{i} .
\end{gathered}
$$

Theorem 3. Suppose that the conditions of Theorem 1 are satisfied. Then

$$
\sup _{x}\left|\mathbf{P}\left(\left(\theta^{*}-\theta\right) / d^{*}<x\right)-\Phi(x)\right| \rightarrow 0 .
$$

Theorem 4. Suppose that the conditions of Theorem 2 are satisfied. Then

$$
\sup _{x}\left|\mathbf{P}\left(\left(\theta^{* *}-\theta\right) / d^{* *}<x\right)-\Phi(x)\right| \rightarrow 0 .
$$

\section{Some Generalizations}

Below we consider a more general problem in which $a_{i}=a_{i}^{(N)}, b_{i}=b_{i}^{(N)}, \sigma_{i}=\sigma_{i}^{N}$ and $X_{i}=X_{i}^{(N)}$, with the superscript emphasizing that the variables may depend on the number $N$ of observations. In order to keep our notation reasonable, we will omit the superscript $(N)$ of the values $a_{i}, b_{i}, \sigma_{i}$, and $X_{i}$.

We need the following notations:

$$
\begin{gathered}
\alpha_{i}=a_{i} b_{i} /\left(1+b_{i} \theta\right), \quad \beta_{i}=\left(1+b_{i} \theta\right) \sigma_{i}, \quad \gamma_{i}=\gamma_{i}(\theta), \quad K_{i}=K_{i}(\theta), \\
A_{c}=\sum c_{i} \alpha_{i}, \quad B_{c}^{2}=\sum c_{i}^{2} \beta_{i}^{2}, \quad d_{c}=B_{c} / A_{c}, \\
A_{\gamma}=\sum \gamma_{i} \alpha_{i}, \quad B_{\gamma}^{2}=\sum \gamma_{i}^{2} \beta_{i}^{\prime} \quad d_{\gamma}=B_{\gamma} / A_{\gamma}, \\
\hat{d}_{c}=B_{c} / \sum c_{i} b_{i} X_{i}, \quad \hat{d}_{\gamma}=B_{\gamma} / \sum \gamma_{i} b_{i} X_{i} .
\end{gathered}
$$

It is easy to see that in this case $d_{c}=d\left(\left\{c_{i}\right\}\right)$ and $d_{\gamma}=d\left(\left\{\gamma_{i}\right\}\right)$.

Theorem 5. Suppose that the condition

$$
\max _{k \leq N} c_{k}^{2} \beta_{k}^{2} / B_{c}^{2} \rightarrow 0
$$


is satisfied. Then

$$
\sup _{x}\left|\mathbf{P}\left(\left(\theta^{*}-\theta\right) / \hat{d}_{c}<x\right)-\Phi(x)\right| \rightarrow 0 .
$$

Theorem 6. Suppose that (3.4) and the condition

$$
\sum c_{i}^{2} b_{i}^{2} \sigma_{i}^{2} / A_{c}^{2} \rightarrow 0
$$

are satisfied. Then the conclusion of Theorem 1 holds.

Theorem 7. Suppose that (3.4), (3.5), and

$$
\sum c_{i}^{2} \alpha_{i}^{2} / A_{c}^{2} \rightarrow 0
$$

are satisfied. Then the conclusion of Theorem 4 holds.

It is easy to see that Theorems 1 and 3 are immediate from Theorems 6 and 7 .

We turn to studying the estimator $\theta^{* *}$. Studying the properties of this estimator, we always assume the following conditions to be satisfied:

$$
\begin{gathered}
d_{c} \rightarrow 0, \\
\max _{k \leq N} \gamma_{k}^{2} \beta_{k}^{2} / B_{\gamma}^{2} \rightarrow 0, \\
d_{c}^{2}\left(\sum \beta_{i}^{2} K_{i}^{2}\right) / B_{\gamma}^{2} \rightarrow 0 .
\end{gathered}
$$

Theorem 8. Suppose that (3.7)-(3.9) are satisfied. Then

$$
\sup _{x}\left|\mathbf{P}\left(\left(\theta^{*}-\theta\right) / \hat{d}_{\gamma}<x\right)-\Phi(x)\right| \rightarrow 0 .
$$

Theorem 9. Suppose that the conditions of Theorem 8 are satisfied and

$$
\begin{gathered}
\sum \gamma_{i}^{2} b_{i}^{2} \sigma_{i}^{2} / A_{\gamma}^{2} \rightarrow 0, \\
d_{c}^{2}\left(\sum b_{i}^{2} \sigma_{i}^{2} K_{i}^{2}\right) / A_{\gamma}^{2} \rightarrow 0, \\
d_{c}\left(\sum \alpha_{i} K_{i}\right) / A_{\gamma} \rightarrow 0 .
\end{gathered}
$$

Then all conclusions of Theorem 2 are valid.

Theorem 10. Suppose that the conditions of Theorem 8 are satisfied and

$$
\begin{gathered}
d_{c}^{4} \sum K_{i}^{2}\left(\alpha_{i}^{2}+b_{i}^{2} \sigma_{i}^{2}\right) / B_{\gamma}^{2} \rightarrow 0 \\
d_{c}^{2} \sum \gamma_{i}^{2}\left(\alpha_{i}^{2}+b_{i}^{2} \sigma_{i}^{2}\right) / B_{\gamma}^{2} \rightarrow 0 .
\end{gathered}
$$

Then the conclusion of Theorem 4 is valid.

It is easy to verify that Theorems 2 and 4 are particular instances of Theorems 9 and 10 .

Remark 6. Studying the estimator $\theta^{* *}$ of the second step, we essentially use the assumption that the estimator $\theta^{*}$ of the first step is consistent. As we see from (3.18) and (3.2), consistency of this estimator is guaranteed by (3.7). Observe that (3.7) is essential: otherwise the estimator $\theta^{*}$ could satisfy the conditions of Theorem 1 but fail to be consistent. Indeed, suppose that $a_{i}=c_{i}=\sigma_{i}=1$ and $b_{i}=1 / i^{1-\varepsilon}$ for $0<\varepsilon<1 / 2$. It is easy to verify that in this case

$$
A_{c} \sim \sum b_{i} \sim N^{\varepsilon} / \varepsilon \rightarrow \infty, \quad d_{c}^{2} \sim N / A_{c}^{2} \sim \varepsilon^{2} N^{1-2 \varepsilon} \rightarrow \infty .
$$

At the same time, all conditions of Theorem 1 are satisfied, because

$$
\sum c_{i}^{2} b_{i}^{2} \sigma_{i}^{2}=\sum i^{2 \varepsilon-2}<\infty
$$




$$
\max _{k \leq N} c_{k}^{2} \beta_{k}^{2}=(1+\theta)^{2}<\infty, \quad \sum c_{i}^{2} \beta_{i}^{2} \sim N \rightarrow \infty .
$$

Remark 7. We present some arguments that enable us to guess the simple form of estimators (1.3) and (1.4). Rewrite (1.1) as

$$
\left(1+b_{i} \theta\right) X_{i}=a_{i}+\beta_{i} \xi_{i} .
$$

Multiplying (3.15) by $c_{i}$ and summing the result over $i$, we come to the following useful identity:

$$
\sum c_{i} X_{i}+\theta \sum c_{i} b_{i} X_{i}=\sum c_{i} a_{i}+\sum c_{i} \beta_{i} \xi_{i}
$$

We may suppose that the weighted sum of the errors of $\xi_{i}$, the last summand in (3.16), is small as compared with the other sums of positive summands. Therefore, it is natural to discard the last summand in (3.16), substituting the estimator $\theta^{*}$ for the unknown parameter $\theta$ in the modified equality. Solving the equation

$$
\sum c_{i} X_{i}+\theta^{*} \sum c_{i} b_{i} X_{i}=\sum c_{i} a_{i},
$$

we find representation (1.3) for the estimator $\theta^{*}$.

Now, subtracting (3.16) from (3.17) and using (1.1), we arrive at

$$
\theta^{*}-\theta=\frac{-\sum c_{i}\left(1+b_{i} \theta\right) \sigma_{i} \xi_{i}}{\sum c_{i} b_{i} X_{i}}=\frac{-\sum c_{i} \beta_{i} \xi_{i}}{\sum c_{i} \alpha_{i}+\sum c_{i} b_{i} \sigma_{i} \xi_{i}} .
$$

Representation (3.18) plays a key role in studying the properties of the estimator $\theta^{*}$.

Observe that, by analogy with (3.18), we have the following representation for the estimator $\theta^{* *}$ :

$$
\theta^{* *}-\theta=\frac{\sum \gamma_{i}\left(\theta^{*}\right) \beta_{i} \xi_{i}}{\sum \gamma_{i}\left(\theta^{*}\right) b_{i} X_{i}}
$$

Remark 8. Throughout the article, $\theta$ is an unknown parameter. Moreover, the values $\left\{\sigma_{i}\right\}$ may be unknown parameters either. Thus, the most of the conditions in all assertions of the article are constraints on the values involving unknown parameters. Clearly, in practical application of these assertions we should check the conditions for all values of all unknown parameters (as, for instance, in [3]).

\section{Proofs of the Properties of the Estimator}

$\theta^{*}$

Proof of Theorem 5. Put

$$
\beta_{i, N}=c_{i} \beta_{i} / B_{c}, \quad \beta(N)=\max _{k \leq N} \beta_{k, N} .
$$

In this case, (3.18) and (3.3) yield the representation

$$
\left(\theta^{*}-\theta\right) / \hat{d}_{c}=-\sum \beta_{i, N} \xi_{i} .
$$

Lemma 1. If (3.4) is satisfied then

$$
\sup _{x}\left|\mathbf{P}\left(-\sum \beta_{i, N} \xi_{i}<x\right)-\Phi(x)\right| \rightarrow 0 .
$$

Proof. This assertion is a particular instance of the central limit theorem for a scheme of series. Therefore, it suffices (see [4, Chapter 8 , Theorem 5]) to verify validity of the condition

$$
D_{2} \equiv \sum \mathbf{E} \min \left\{\left(\beta_{i, N} \xi_{i}\right)^{2},\left|\beta_{i, N} \xi_{i}\right|^{3}\right\} \rightarrow 0 .
$$


From the definition (4.1) of $\beta(N)$ we obtain

$$
D_{2} \leq \sum \beta_{i, N}^{2} \mathbf{E} \min \left\{\xi_{i}^{2}, \beta(N)\left|\xi_{i}\right|^{3}\right\}=\mathbf{E} \min \left\{\xi_{1}^{2}, \beta(N)\left|\xi_{1}\right|^{3}\right\} .
$$

In (4.4) we have used the fact that $\sum \beta_{i, N}^{2}=1$ by (4.1). Now, to derive (4.3) from (4.4), it suffices to observe that $\beta(N) \rightarrow 0$, because $\beta(N)$ coincides with the left-hand side of (3.4) by definition.

The claim of Theorem 5 is immediate from (4.2) and Lemma 1.

We turn to proving Theorem 6 . We use the representation

$$
\frac{\theta^{*}-\theta}{d_{c}}=\frac{-\sum \beta_{i, N} \xi_{i}}{1+\sum c_{i} b_{i} \sigma_{i} \xi_{i} / A_{c}}
$$

which follows from (2.2) and (3.18).

Lemma 2. If (3.5) is satisfied then $\sum c_{i} b_{i} \sigma_{i} \xi_{i} / A_{c} \stackrel{p}{\rightarrow} 0$.

This assertion follows from Chebyshev's inequality, since

$$
\mathbf{E}\left(\sum c_{i} b_{i} \sigma_{i} \xi_{i} / A_{c}\right)=0
$$

and the variance of this expression coincides with the left-hand side of (3.5).

The claim of Theorem 6 is immediate from (4.5), Lemma 2, and Theorem 5.

Now, we turn to proving Theorem 7 . We first prove two auxiliary assertions:

Lemma 3. Suppose that (3.4) is satisfied. Then

$$
\sum c_{i}^{2} \beta_{i}^{2} \xi_{i}^{2} / \sum c_{i}^{2} \beta_{i}^{2} \stackrel{p}{\rightarrow} 1
$$

Proof. Using (4.1), we can rewrite the claim of the lemma as

$$
\sum \beta_{i, N}^{2}\left(\xi_{i}^{2}-1\right) \stackrel{p}{\rightarrow} 0, \quad \mathbf{E}\left(\xi_{i}^{2}-1\right)=0 .
$$

However, the convergence in (4.7) is a particular instance of the law of large numbers for a scheme of series (see [4, Chapter 8, Theorem 3]). Therefore, to validate (4.7), it suffices to show that the following condition is satisfied:

$$
D_{1} \equiv \sum \mathbf{E} \min \left\{\beta_{i, N}^{4}\left(\xi_{i}^{2}-1\right)^{2} ;\left|\beta_{i, N}^{2}\left(\xi_{i}^{2}-1\right)\right|\right\} \rightarrow 0
$$

We have

$D_{1} \leq \sum \beta_{i, N}^{2} \mathbf{E} \min \left\{\beta^{2}(N)\left(\xi_{i}^{2}-1\right)^{2} ;\left|\xi_{i}^{2}-1\right|\right\}=\mathbf{E} \min \left\{\beta^{2}(N)\left(\xi_{1}^{2}-1\right)^{2} ;\left|\xi_{1}^{2}-1\right|\right\}$.

Deriving (4.9), we have used the fact that $\sum \beta_{i, N}^{2}=1$ in view of (4.1). Since $\beta(N) \rightarrow 0$ by (3.4), from (4.9) we now obtain (4.8), which proves the sought assertion (4.7).

Denote

$$
\delta^{*}=\left(\sum c_{i}^{2} \beta_{i}^{* 2}\right)^{1 / 2}-\left(\sum c_{i}^{2} \beta_{i}^{2} \xi_{i}^{2}\right)^{1 / 2}, \quad \delta_{c}=\left(\sum c_{i}^{2} \beta_{i}^{2} \xi_{i}^{2}\right)^{1 / 2}-\left(\sum c_{i}^{2} \beta_{i}^{2}\right)^{1 / 2} .
$$

Lemma 4. Under the conditions of Theorem 7,

$$
\delta_{c} / B_{c} \stackrel{p}{\rightarrow} 0, \quad \delta^{*} / B_{c} \stackrel{p}{\rightarrow} 0 .
$$

Proof. The first claim of the lemma following from Lemma 3, it suffices to prove the second. Observe that

$$
\beta_{i}^{*}-\beta_{i} \xi_{i}=\left(1+b_{i} \theta^{*}\right) X_{i}-a_{i}-\left(1+b_{i} \theta\right) \sigma_{i} \xi_{i}=\left(\theta^{*}-\theta\right) b_{i} X_{i} .
$$


Furthermore as soon as, the root of the sum of squares possesses all properties of a norm, we have

$$
\left|\delta^{*}\right| \leq\left(\sum c_{i}^{2}\left(\beta_{i}^{*}-\beta_{i} \xi_{i}\right)^{2}\right)^{1 / 2}=\left|\theta^{*}-\theta\right| \delta_{0 c}
$$

where $\delta_{0 c}=\left(\sum c_{i}^{2} b_{i}^{2} X_{i}^{2}\right)^{1 / 2}$. Therefore

$$
\frac{\left|\delta^{*}\right|}{B_{c}} \leq \frac{\left|\theta^{*}-\theta\right|}{d_{c}} \frac{\delta_{0 c}}{A_{c}}
$$

Since $\mathbf{E} \delta_{0 c}^{2}=\sum c_{i}^{2} \alpha_{i}^{2}+\sum c_{i}^{2} b_{i}^{2} \sigma_{i}^{2}$, by (3.5) and (3.6) the second factor in (4.11) vanishes in probability. Using this fact and asymptotic normality of the estimator $\theta^{*}$, from (4.11) we easily deduce the second claim of the lemma.

Now, we complete the proof of Theorem 7. Using (3.18) and the notations (3.2), (4.1), and (4.10), we obtain

$$
\frac{\theta^{*}-\theta}{d^{*}}=\frac{-\sum c_{i} \beta_{i} \xi_{i}}{\left(\sum c_{i}^{2} \beta_{i}^{* 2}\right)^{1 / 2}}=\frac{-\sum \beta_{i, N} \xi_{i}}{1+\delta^{*} / B_{c}+\delta_{c} / B_{c}} .
$$

Now, Theorem 7 is immediate from (4.12) and Lemmas 1 and 4 .

\section{Proof of Theorem 8}

Fix $\theta>0$, preserving the above notations. Put

$$
q_{i}=-c_{i} \sigma_{i} / A_{c}, \quad r_{i}=c_{i} b_{i} \sigma_{i} / A_{c}, \quad Z_{1}=-\sum q_{i} \xi_{i}, \quad Z_{2}=\sum r_{i} \xi_{i} .
$$

Then by (3.18)

$$
\theta^{*}=\left(\theta+Z_{1}\right) /\left(1+Z_{2}\right)
$$

Introduce the random variable

$$
\tilde{\theta}=\min \left\{2 \theta, \theta+Z_{1}\right\} / \max \left\{1 / 2,1+Z_{2}\right\} .
$$

Lemma 5. If (3.7) is satisfied then

$$
\mathbf{P}\left(\tilde{\theta} \neq \theta^{*}\right) \rightarrow 0
$$

Proof. By (1.2) and (5.1) we have $\mathbf{E} Z_{1}=\mathbf{E} Z_{2}=0$; therefore,

$$
\begin{gathered}
\mathbf{E}\left(Z_{1}\right)^{2}=\sum c_{i}^{2} \sigma_{i}^{2} / A_{c}^{2} \leq B_{c}^{2} / A_{c}^{2}=d_{c}^{2} \rightarrow 0, \\
\mathbf{E}\left(Z_{2}\right)^{2}=\sum c_{i}^{2} b_{i}^{2} \sigma_{i}^{2} / A_{c}^{2} \leq B_{c}^{2} / A_{c}^{2} \theta^{2}=d_{c}^{2} / \theta^{2} \rightarrow 0
\end{gathered}
$$

by (3.7). We infer in particular that $Z_{1} \stackrel{p}{\rightarrow} 0$ and $Z_{2} \stackrel{p}{\rightarrow} 0$. Thus, to complete the proof of Lemma 5, we need to compare (5.2) and (5.3), observing that

$$
\mathbf{P}\left(\tilde{\theta} \neq \theta^{*}\right) \leq \mathbf{P}\left(Z_{2}<-1 / 2\right)+\mathbf{P}\left(Z_{1}>\theta\right) \rightarrow 0 .
$$

Put

$$
\begin{aligned}
\tilde{\gamma}_{i}=\gamma_{i}(\tilde{\theta}), \quad Z_{3} & =\sum\left(\tilde{\gamma}_{i}-\gamma_{i}\right) \beta_{i} \xi_{i}, \quad \tilde{d}_{\gamma}=B_{\gamma} / \sum \tilde{\gamma}_{i} b_{i} X_{i}, \\
\tilde{\theta}^{* *} & =\sum \tilde{\gamma}_{i}\left(a_{i}-X_{i}\right) / \sum \tilde{\gamma}_{i} b_{i} X_{i} .
\end{aligned}
$$

From (1.4), (5.6), (5.7), and Lemma 5 we immediately see that

$$
\mathbf{P}\left(\tilde{\theta}^{* *} \neq \theta^{* *}\right) \rightarrow 0, \quad \mathbf{P}\left(\tilde{d}_{\gamma} \neq \hat{d}_{\gamma}\right) \rightarrow 0 .
$$


Using (1.4), (3.19), (5.6), and (5.7), we obtain

$$
\left(\tilde{\theta}^{* *}-\theta\right) / \tilde{d}_{\gamma}=-\sum \tilde{\gamma}_{i} \beta_{i} \xi_{i} / B_{\gamma}=-\sum \gamma_{i} \beta_{i} \xi_{i} / B_{\gamma}+Z_{3} / B_{\gamma} .
$$

Formula (5.9) is central to the proof of Theorem 8. Also, we need the notations

$$
\tilde{\theta}_{j}=\frac{\min \left\{2 \theta, \theta+\sum_{i \neq j} q_{i} \xi_{i}\right\}}{\max \left\{1 / 2,1+\sum_{i \neq j} r_{i} \xi_{i}\right\}}, \quad \tilde{\theta}_{j k}=\frac{\min \left\{2 \theta, \theta+\sum_{i \neq j, k} q_{i} \xi_{i}\right\}}{\max \left\{1 / 2,1+\sum_{i \neq j, k} r_{i} \xi_{i}\right\}} .
$$

Lemma 6. For each $j$, the value $\tilde{\theta}_{j}$ is independent of $\xi_{j}$ and

$$
\left|\tilde{\theta}_{j}-\tilde{\theta}\right| \leq \widetilde{K}_{j}\left|\xi_{j}\right| \quad \text { for } \quad \widetilde{K}_{j}=8\left(\left|q_{j}\right|+\theta r_{j}\right)=8 c_{j} \beta_{j} / A_{c} .
$$

Proof. The independence follows from the definition of $\tilde{\theta}_{j}$. Now, fix a number $j$ and all values $\xi_{i}$ for $i \neq j$. Put

$$
\begin{gathered}
q=\theta+\sum_{i \neq j} q_{i} \xi_{i}, \quad r=1+\sum_{i \neq j} r_{i} \xi_{i}, \\
f_{1}\left(\xi_{j}\right)=\min \left\{2 \theta, q+q_{j} \xi_{j}\right\}, \quad f_{2}\left(\xi_{j}\right)=\max \left\{1 / 2,1+r+r_{j} \xi_{j}\right\} .
\end{gathered}
$$

With these notations, we have $\tilde{\theta}=f_{1}\left(\xi_{j}\right) / f_{2}\left(\xi_{j}\right)$ and $\tilde{\theta}_{j}=f_{1}(0) / f_{2}(0)$. Therefore,

$$
\tilde{\theta}-\tilde{\theta}_{j}=\frac{f_{1}\left(\xi_{j}\right)-f_{1}(0)}{f_{2}\left(\xi_{j}\right)}+f_{1}(0) \frac{f_{2}(0)-f_{2}\left(\xi_{j}\right)}{f_{2}\left(\xi_{j}\right) f_{2}(0)} .
$$

It is clear that

$$
\left|f_{1}\left(\xi_{j}\right)-f_{1}(0)\right| \leq\left|q_{j}\right|\left|\xi_{j}\right|, \quad\left|f_{2}\left(\xi_{j}\right)-f_{2}(0)\right| \leq r_{j}\left|\xi_{j}\right|, \quad\left|f_{1}(\cdot)\right| \leq 2 \theta, \quad\left|f_{2}(\cdot)\right| \geq 1 / 2 .
$$

Inserting these relations in (5.10), we obtain

$$
\left|\tilde{\theta}-\tilde{\theta}_{j}\right| \leq \frac{\left|q_{j}\right|\left|\xi_{j}\right|}{1 / 2}+2 \theta \frac{r_{j}\left|\xi_{j}\right|}{1 / 4} \leq 8\left(\left|q_{j}\right|+\theta r_{j}\right)\left|\xi_{j}\right| .
$$

Lemma 7. For all $j \neq k$, the value $\tilde{\theta}_{j k}$ is independent of $\xi_{j}$ and $\xi_{k}$, and

$$
\left|\tilde{\theta}_{j k}-\tilde{\theta}_{k}\right| \leq \widetilde{K}_{j}\left|\xi_{j}\right| .
$$

Proof. The proof of the lemma repeats that of Lemma 6 on taking $\xi_{k}$ to be identically zero.

Lemma 8. The following inequality is valid:

$$
\mathbf{E}|\tilde{\theta}-\theta|^{2} \leq 16 d_{c}^{2}
$$

Proof. By analogy with Lemma 6, we put

$$
f_{1}\left(Z_{1}\right)=\min \left\{2 \theta, \theta+Z_{1}\right\}, \quad f_{2}\left(Z_{2}\right)=\max \left\{1 / 2,1+Z_{2}\right\} .
$$

It is clear that $f_{1}(0)=\theta, f_{2}(0)=1,\left|f_{2}\left(Z_{2}\right)\right| \geq 1 / 2$, and

$$
\left|f_{1}\left(Z_{1}\right)-f_{1}(0)\right| \leq\left|Z_{1}\right|, \quad\left|f_{2}\left(Z_{2}\right)-f_{2}(0)\right| \leq\left|Z_{2}\right| .
$$


Therefore,

$$
\begin{aligned}
|\tilde{\theta}-\theta|=\mid \frac{f_{1}\left(Z_{1}\right)}{f_{2}\left(Z_{2}\right)} & -\frac{f_{1}(0)}{f_{2}(0)}|=| \frac{f_{1}\left(Z_{1}\right)-f_{1}(0)}{f_{2}\left(Z_{2}\right)}+f_{1}(0) \frac{f_{2}(0)-f_{2}\left(Z_{2}\right)}{f_{2}\left(Z_{2}\right) f_{2}(0)} \mid \\
& \leq \frac{\left|Z_{1}\right|}{1 / 2}+\theta \frac{\left|Z_{2}\right|}{1 / 2}=2\left|Z_{1}\right|+2 \theta\left|Z_{2}\right| .
\end{aligned}
$$

Consequently,

$$
\mathbf{E}|\tilde{\theta}-\theta|^{2} \leq 2 \mathbf{E}\left(2\left|Z_{1}\right|\right)^{2}+2 \mathbf{E}\left(2 \theta\left|Z_{2}\right|\right)^{2}
$$

Inserting the estimates of (5.4) and (5.5) in the last inequality, we deduce the claim.

Lemma 9. For all $i$ we have

$$
\mathbf{E}\left|\tilde{\theta}_{i}-\theta\right|^{2} \leq 16 d_{c}^{2}
$$

Proof. The proof of the lemma repeats verbatim that of Lemma 8 on taking $\xi_{i}$ to be identically zero.

Put $\tilde{\gamma}_{i i}=\gamma_{i}\left(\tilde{\theta}_{i}\right)$.

Lemma 10. The following inequality is valid:

$$
\Delta_{1} \equiv \mathbf{E}\left|\sum \beta_{i}\left(\tilde{\gamma}_{i}-\tilde{\gamma}_{i i}\right) \xi_{i}\right| \leq 8 d_{c}\left(\sum \beta_{i}^{2} K_{i}^{2}\right)^{1 / 2}
$$

Proof. By (2.3) and Lemma 6, we have

$$
\left|\tilde{\gamma}_{i}-\tilde{\gamma}_{i i}\right| \leq K_{i}\left|\tilde{\theta}-\tilde{\theta}_{i}\right| \leq K_{i} \widetilde{K}_{i}\left|\xi_{i}\right|
$$

Consequently,

$$
\Delta_{1} \leq \mathbf{E} \sum \beta_{i} K_{i} \widetilde{K}_{i} \xi_{i}^{2}=\sum \beta_{i} K_{i} \widetilde{K}_{i}
$$

Using the definition of the constants $\left\{\widetilde{K}_{i}\right\}$, we now obtain

$$
\left(\sum \beta_{i} K_{i} \widetilde{K}_{i}\right)^{2} \leq \sum \beta_{i}^{2} K_{i}^{2} \sum \widetilde{K}_{i}^{2}=\left(8 d_{c}\right)^{2} \sum \beta_{i}^{2} K_{i}^{2}
$$

Inequalities (5.11) and (5.12) yield the claim of Lemma 10.

Lemma 11. The following inequality is valid:

$$
\Delta_{2} \equiv \mathbf{E}\left(\sum \beta_{i}\left(\tilde{\gamma}_{i i}-\gamma_{i}\right) \xi_{i}\right)^{2} \leq 80 d_{c}^{2}\left(\sum \beta_{i}^{2} K_{i}^{2}\right)
$$

Proof. Put $\delta_{i i}=\tilde{\gamma}_{i i}-\gamma_{i}$. Clearly, $\delta_{i i}$ is independent of $\xi_{i}$. Therefore,

$$
\begin{gathered}
\mathbf{E}\left(\sum \beta_{i} \delta_{i i} \xi_{i}\right)^{2}=\sum \beta_{i}^{2} \mathbf{E} \delta_{i i}^{2} \xi_{i}^{2}+\sum_{i \neq j} \beta_{i} \beta_{j} \mathbf{E} \delta_{i i} \xi_{i} \delta_{j j} \xi_{j} \\
=\sum \beta_{i}^{2} \mathbf{E} \delta_{i i}^{2}+\sum_{i \neq j} \beta_{i} \beta_{j} \mathbf{E} \delta_{i i} \xi_{i} \delta_{j j} \xi_{j} .
\end{gathered}
$$

Using (2.3) and Lemma 9, we obtain

$$
\sum \beta_{i}^{2} \mathbf{E} \delta_{i i}^{2} \leq \sum \beta_{i}^{2} \mathbf{E} K_{i}^{2}\left|\tilde{\theta}_{i}-\theta\right|^{2} \leq 16 d_{c}^{2}\left(\sum \beta_{i}^{2} K_{i}^{2}\right) .
$$

Denote $\tilde{\gamma}_{i i j}=\gamma_{i}\left(\tilde{\theta}_{i j}\right), \delta_{i i j}=\tilde{\gamma}_{i i j}-\gamma_{i}$, and $\tilde{\delta}_{i i j}=\tilde{\gamma}_{i i}-\tilde{\gamma}_{i i j}$. Then

$$
\delta_{i i}=\delta_{i i j}+\tilde{\delta}_{i i j}
$$


moreover, $\tilde{\delta}_{i i j}$ is independent of $\xi_{i}$ and by Lemma 7 and (2.3)

$$
\left|\tilde{\delta}_{i i j}\right| \leq K_{i}\left|\tilde{\theta}_{i}-\tilde{\theta}_{i j}\right| \leq K_{i} \widetilde{K}_{j}\left|\xi_{j}\right| .
$$

Inserting (5.15) in the second summand of (5.13), we obtain

$$
\begin{gathered}
\sum_{i \neq j} \beta_{i} \beta_{j} \mathbf{E} \delta_{i i} \xi_{i} \delta_{j j} \xi_{j}=\sum_{i \neq j} \beta_{i} \beta_{j} \mathbf{E}\left(\delta_{i i j}+\tilde{\delta}_{i i j}\right) \xi_{i}\left(\delta_{j j i}+\tilde{\delta}_{j j i}\right) \xi_{j} \\
=\sum_{i \neq j} \beta_{i} \beta_{j} \mathbf{E} \delta_{i i j} \delta_{j j i} \xi_{i} \xi_{j}+\sum_{i \neq j} \beta_{i} \beta_{j} \mathbf{E} \delta_{i i j} \tilde{\delta}_{j j i} \xi_{i} \xi_{j} \\
+\sum_{i \neq j} \beta_{i} \beta_{j} \mathbf{E} \delta_{j j i} \tilde{\delta}_{i i j} \xi_{i} \xi_{j}+\sum_{i \neq j} \beta_{i} \beta_{j} \mathbf{E} \tilde{\delta}_{i i j} \tilde{\delta}_{j j i} \xi_{i} \xi_{j} \\
\leq 0+0+0+\sum_{i \neq j} \beta_{i} \beta_{j} \mathbf{E} K_{i} \widetilde{K}_{i} K_{j} \widetilde{K}_{j} \xi_{i}^{2} \xi_{j}^{2} \\
\leq\left(\sum \beta_{i} K_{i} \widetilde{K}_{i}\right)^{2} \leq 64 d_{c}^{2}\left(\sum \beta_{i}^{2} K_{i}^{2}\right) .
\end{gathered}
$$

Deriving the last relation, we have used (5.12).

The claim of the lemma ensues from (5.14), (5.16), and (5.13).

Lemma 12. The following inequality is valid:

$$
\Delta_{3} \equiv \mathbf{E}\left|Z_{3}\right| \leq 17 d_{c}\left(\sum \beta_{i}^{2} K_{i}^{2}\right)^{1 / 2} .
$$

To prove this assertion, it suffices to observe that $\Delta_{3} \leq \Delta_{1}+\Delta_{2}^{1 / 2}$ and use Lemmas 10 and 11.

Lemma 13. If (3.8) is satisfied then

$$
\sup _{x}\left|\mathbf{P}\left(-\sum \gamma_{i} \beta_{i} \xi_{i} / B_{\gamma}<x\right)-\Phi(x)\right| \rightarrow 0 .
$$

To obtain this convergence, it suffices to repeat the derivation of Lemma 2 for $\beta_{i, N}=-\gamma_{i} \beta_{i} / B_{\gamma}$.

We now complete the proof of Theorem 8. It follows from Lemma 12 and (3.9) that

$$
Z_{3} / B_{\gamma} \stackrel{p}{\rightarrow} 0
$$

Therefore, the claim of Theorem 8 ensues from (5.9), (5.18), and Lemma 13.

\section{Proofs of Theorems 9 And 10}

Denote

$$
Z_{4}=\sum\left(\tilde{\gamma}_{i}-\gamma_{i}\right) \alpha_{i}, \quad Z_{5}=\sum \gamma_{i} b_{i} \sigma_{i} \xi_{i}, \quad Z_{6}=\sum\left(\tilde{\gamma}_{i}-\gamma_{i}\right) b_{i} \sigma_{i} \xi_{i} .
$$

Using (1.4), (3.19), and the notations (3.2), (5.6), and (5.7), we obtain

$$
\frac{\tilde{\theta}^{* *}-\theta}{d\left(\left\{\gamma_{i}\right\}\right)}=\frac{-\sum \tilde{\gamma}_{i} \beta_{i} \xi_{i}}{\sum \tilde{\gamma}_{i} b_{i} X_{i}} \cdot \frac{A_{\gamma}}{B_{\gamma}}=\frac{-\sum \gamma_{i} \beta_{i} \xi_{i} / B_{\gamma}+Z_{3} / B_{\gamma}}{1+\left(Z_{4}+Z_{5}+Z_{6}\right) / A_{\gamma}} .
$$

Representation (6.2) plays a key role in the proof of Theorem 9.

Lemma 14. The following estimate holds:

$$
\Delta_{6} \equiv \mathbf{E}\left|Z_{6}\right| \leq 17 d_{c}\left(\sum b_{i}^{2} \sigma_{i}^{2} K_{i}^{2}\right)^{1 / 2} .
$$


To derive this inequality, it suffices to repeat the proof of Lemmas 10-12 with $b_{i} \sigma_{i}$ substituted for $\beta_{i}$.

Lemma 15. The following estimate is valid:

$$
\Delta_{4} \equiv \mathbf{E}\left|Z_{4}\right| \leq 4 d_{c}\left(\sum \alpha_{i} K_{i}\right)
$$

Proof. By (2.3) we have $\left|\tilde{\gamma}_{i}-\gamma_{i}\right| \leq K_{i}|\tilde{\theta}-\theta|$. Using Lemma 8, we obtain

$$
\mathbf{E}\left|\tilde{\gamma}_{i}-\gamma_{i}\right| \leq K_{i}\left(\mathbf{E}(\tilde{\theta}-\theta)^{2}\right)^{1 / 2} \leq(16)^{1 / 2} d_{c} K_{i}
$$

Inserting this inequality in the definition (6.1) of $Z_{4}$, we arrive at the claim of the lemma.

Lemma 16. The following estimate is valid:

$$
\Delta_{5} \equiv \mathbf{E}\left|Z_{5}\right| \leq\left(\sum \gamma_{i}^{2} b_{i}^{2} \sigma_{i}^{2}\right)^{1 / 2}
$$

To prove this inequality, it suffices to note that $\mathbf{E} Z_{5}=0$ and $\mathbf{D} Z_{5}=\sum \gamma_{i}^{2} b_{i}^{2} \sigma_{i}^{2}$ in view of (1.2) and (6.1).

Now, we complete the proof of Theorem 9. It follows from Lemmas 14-16 and (3.10)-(3.12) that $\mathbf{E}\left|Z_{4}+Z_{5}+Z_{6}\right| / A_{\gamma} \rightarrow 0$; therefore,

$$
\left(Z_{4}+Z_{5}+Z_{6}\right) / A_{\gamma} \stackrel{p}{\rightarrow} 0 .
$$

The claim of the theorem ensues now from (6.2), (6.3), (5.18), and Lemma 13.

We turn to proving Theorem 10. Put

$$
\begin{gathered}
\tilde{\beta}_{i}=\left(1+b_{i} \tilde{\theta}\right) X_{i}-a_{i}, \quad \tilde{d}^{* *}=\left(\sum \tilde{\gamma}_{i}^{2} \tilde{\beta}_{i}^{2}\right)^{1 / 2} / \sum \tilde{\gamma}_{i} b_{i} X_{i}, \\
\delta=\left(\sum \tilde{\gamma}_{i}^{2} \tilde{\beta}_{i}^{2}\right)^{1 / 2}-\left(\sum \gamma_{i}^{2} \beta_{i}^{2} \xi_{i}^{2}\right)^{1 / 2}, \quad \delta_{0}=\left(\sum \gamma_{i}^{2} \beta_{i}^{2} \xi_{i}^{2}\right)^{1 / 2}-\left(\sum \gamma_{i}^{2} \beta_{i}^{2}\right)^{1 / 2}
\end{gathered}
$$

and note that

$$
\mathbf{P}\left(\tilde{d}^{* *} \neq d^{* *}\right) \rightarrow 0
$$

by Lemma 5 and (2.9), (5.6), and (6.4). Using (3.19) and the notations (1.4), (5.6), (5.7), (6.4), and (6.5), we easily obtain the formula

$$
\frac{\tilde{\theta}^{* *}-\theta}{\tilde{d}^{* *}}=\frac{-\sum \tilde{\gamma}_{i} \beta_{i} \xi_{i}}{\left(\sum \tilde{\gamma}_{i}^{2} \tilde{\beta}_{i}^{2}\right)^{1 / 2}}=\frac{-\sum \gamma_{i} \beta_{i} \xi_{i} / B_{\gamma}+Z_{3} / B_{\gamma}}{1+\left(\delta+\delta_{0}\right) / B_{\gamma}} .
$$

This formula plays a key role in the proof of Theorem 10.

Denote

$$
\delta_{0 \beta}=\left(\sum K_{i}^{2} \beta_{i}^{2} \xi_{i}^{2}\right)^{1 / 2}, \quad \delta_{0 \gamma}=\left(\sum \gamma_{i}^{2} b_{i}^{2} X_{i}^{2}\right)^{1 / 2}, \quad \delta_{0 K}=\left(\sum K_{i}^{2} b_{i}^{2} X_{i}^{2}\right)^{1 / 2} .
$$

Lemma 17. The following inequality is valid:

$$
|\delta| \leq(\tilde{\theta}-\theta)^{2} \delta_{0 K}+|\tilde{\theta}-\theta|\left(\delta_{0 \gamma}+\delta_{0 \beta}\right) .
$$


Proof. From the properties of a norm we obtain

$$
\begin{gathered}
|\delta| \leq\left(\sum\left(\tilde{\gamma}_{i} \tilde{\beta}_{i}-\gamma_{i} \beta_{i} \xi_{i}\right)^{2}\right)^{1 / 2} \\
=\left(\sum\left(\left(\tilde{\gamma}_{i}-\gamma_{i}\right)\left(\tilde{\beta}_{i}-\beta_{i} \xi_{i}\right)+\gamma_{i}\left(\tilde{\beta}_{i}-\beta_{i} \xi_{i}\right)+\beta_{i} \xi_{i}\left(\tilde{\gamma}_{i}-\gamma_{i}\right)\right)^{2}\right)^{1 / 2} \leq \delta_{\beta \gamma}+\delta_{\beta}+\delta_{\gamma},
\end{gathered}
$$

where

$$
\begin{gathered}
\delta_{\beta \gamma}=\left(\sum\left(\tilde{\gamma}_{i}-\gamma_{i}\right)^{2}\left(\tilde{\beta}_{i}-\beta_{i} \xi_{i}\right)^{2}\right)^{1 / 2} \\
\delta_{\beta}=\left(\sum \gamma_{i}^{2}\left(\tilde{\beta}_{i}-\beta_{i} \xi_{i}\right)^{2}\right)^{1 / 2}, \quad \delta_{\gamma}=\left(\sum\left(\tilde{\gamma}_{i}-\gamma_{i}\right)^{2} \beta_{i}^{2} \xi_{i}^{2}\right)^{1 / 2}
\end{gathered}
$$

Using the identity

$$
\tilde{\beta}_{i}-\beta_{i} \xi_{i}=(\tilde{\theta}-\theta)\left(\alpha_{i}+b_{i} \sigma_{i} \xi_{i}\right)=(\tilde{\theta}-\theta) b_{i} X_{i}
$$

and (2.3), we easily infer that

$$
\delta_{\beta}=|\tilde{\theta}-\theta| \delta_{0 \gamma}, \quad \delta_{\gamma} \leq|\tilde{\theta}-\theta| \delta_{0 \beta}, \quad \delta_{\beta \gamma} \leq|\tilde{\theta}-\theta|^{2} \delta_{0 K}
$$

From (6.9) and (6.10) we obtain the claim of the lemma.

Lemma 18. If the conditions of Theorem 10 are satisfied then $\delta / B_{\gamma} \stackrel{p}{\rightarrow} 0$.

Proof. Since $(|u|+|v|)^{1 / 2} \leq|u|^{1 / 2}+|v|^{1 / 2}$, from Schwarz's inequality and Lemma 17 we find that

$$
\mathbf{E}|\delta|^{1 / 2} \leq\left(\mathbf{E}(\tilde{\theta}-\theta)^{2}\right)^{1 / 2}\left(\mathbf{E} \delta_{0 K}^{2}\right)^{1 / 4}+\left(\mathbf{E}(\tilde{\theta}-\theta)^{2}\right)^{1 / 4}\left(\left(\mathbf{E} \delta_{0 \gamma}^{2}\right)^{1 / 4}+\left(\mathbf{E} \delta_{0 \beta}^{2}\right)^{1 / 4}\right) .
$$

Using (6.8), from the above relation and Lemma 8 we derive

$$
\begin{gathered}
\mathbf{E}|\delta|^{1 / 2} \leq\left(16 d_{c}^{2}\right)^{1 / 2}\left(\sum K_{i}^{2}\left(\alpha_{i}^{2}+b_{i}^{2} \sigma_{i}^{2}\right)\right)^{1 / 4} \\
+\left(16 d_{c}^{2}\right)^{1 / 4}\left(\sum \gamma_{i}^{2}\left(\alpha_{i}^{2}+b_{i}^{2} \sigma_{i}^{2}\right)\right)^{1 / 4}+\left(16 d_{c}^{2}\right)^{1 / 4}\left(\sum K_{i}^{2} \beta_{i}^{2}\right)^{1 / 4} .
\end{gathered}
$$

Dividing the so-obtained expression by $B_{\gamma}^{1 / 2}$ and applying (3.13), (3.14), and (3.9), we easily see that $\mathbf{E}\left(\delta / B_{\gamma}\right)^{1 / 2} \rightarrow 0$; whence the claim of the lemma follows.

Lemma 19. If (3.8) is satisfied then $\delta_{0} / B_{\gamma} \stackrel{p}{\rightarrow} 0$.

To prove this assertion, we have to repeat the derivation of Lemma 3 with the values $c_{i}$ replaced by $\gamma_{i}$.

We now finish the proof of Theorem 10. According to Lemmas 12 and 13, the distribution of the numerator in (6.7) converges to the standard normal distribution. The denominator in (6.7) converges in probability to 1 by Lemmas 18 and 19. To complete the proof, it remains to observe that $\mathbf{P}\left(\tilde{\theta}^{* *} \neq \theta^{* *}\right) \rightarrow 0$ and $\mathbf{P}\left(\tilde{d}^{* *} \neq\right.$ $\left.d^{* *}\right) \rightarrow 0$ in view of (5.8), (6.6) and (3.7). 


\section{REFERENCES}

[1] Draper N. R. and Smith H. Applied Regression Analysis. Statistika, Moscow, (Russian translation), 1973.

[2] Cornish-Bowden A. Basic Mathematics for Biochemists. Mir, Moscow, (Russian translation), 1983.

[3] Borovkov A. A. Mathematical Statistics. Nauka, Moscow, (in Russian), 1984.

[4] Borovkov A. A. Probability. Nauka, Moscow, (in Russian), 1986.

NOVOSIBIRSK 